\title{
On the Appearance of a Cooper Minimum in the Photoionization Cross Sections of the Plasma-Embedded Li Atom
}

\author{
Satyabrata Sahoo ${ }^{1,2}$ and Y. K. Ho ${ }^{1}$ \\ ${ }^{1}$ Institute of Atomic and Molecular Sciences, Academia Sinica, P.O. Box 23-166, Taipei-106, Taiwan, China \\ ${ }^{2}$ Department of Physics, Raidighi College, South 24 Parganas, West Bengal, Pin-743383, India \\ Correspondence should be addressed to Satyabrata Sahoo, sahoo@pub.iams.sinica.edu.tw and \\ Y. K. Ho, ykho@pub.iams.sinica.edu.tw \\ Received 13 April 2009; Accepted 14 June 2009 \\ Recommended by Eric G. Blackman
}

The plasma screening effect is found to uncover a Cooper minimum in the photoionization cross sections from the ground state of the $\mathrm{Li}$ atom embedded in Debye plasma environment. The variation of the location of this minimum with Debye screening length is discussed and analyzed in terms of the instability of the ground state.

Copyright ( $) 2009$ S. Sahoo and Y. K. Ho. This is an open access article distributed under the Creative Commons Attribution License, which permits unrestricted use, distribution, and reproduction in any medium, provided the original work is properly cited.

One of the features of the photoionization process which is most sensitive to the details of interaction, and on which we therefore focus in the present paper, is the plasma screening effect on the so-called Copper minimum. Quite a number of such minima have been found, both theoretically and experimentally [1-14] in ground states of atoms with $Z>10$. Despite this wealth of data, there has been no systematic study of their behavior in the plasma environment. Over the past few decades, considerable interest has been grown in the study of atomic processes in plasma environments [15-26]. The plasma environments can be expected to significantly influence atomic processes primarily through the screening of the long range electrostatic interactions of charged particles. In the investigation of plasma consisting of atomic ions and electrons, a good understanding of the interaction potential among these species is essential.

In the non-relativistic dipole approximation, which is excellent for photoionization cross section below $\hbar \nu \sim 10 \mathrm{keV}$, the cross section is proportional to a weighted sum of the absolute squares of the $l \rightarrow l+1$ and $l \rightarrow l-1$ dipole matrix elements (only a single $l \rightarrow l+1$ in the case of $s$-electron photoionization). An extremely interesting feature of the $l \rightarrow l+1$ dipole matrix element is the existence of zeros in large number of cases. The existence of zeros in dipole matrix elements and the associated minimum (called Cooper minimum) in photoionization cross sections has been known for ground states of alkali atoms for long time. This was first discovered experimentally by Dichtburn et al. [1]. The basic explanation of this phenomenon in alkali-metal atoms was given by Bates [2] and later by Seaton [3] and Cooper $[4,5]$. These Cooper minima occur in the photoionization of outer (and near outer) $n l$ electrons, provided that the discrete $n l$ wave function has at least one node. To account for this behavior, it is observed that the photoionization cross sections from atomic sub-shells whose radial wave functions are node-less have, in general, a different spectral shape than those from sub-shells whose radial wave functions have nodes. Typically, a zero-energy continuum wave function will contain as many antinodes in the inner region as there are occupied sub-shells of the atom corresponding to the value of $l$. With increasing energy, nodes of the continuum wave function will move to smaller value of $r$. For the initial and final state wave functions which have nodes inside the atom, the values of the matrix elements, and hence the cross sections, will depend on the relative positions of these nodes at a particular energy.

Before presenting the plasma screening effect, however, it is useful to discuss the general characteristics of Cooper minima. Generally both discrete and continuum states have wave functions which are oscillatory. Thus the profile of the spectral distribution of the photoionization cross section hinges then on the dependence of the matrix element of 
the initial and final states. Increasing the principal quantum number, the number of nodes in the discrete state increases in accordance with its orbital quantum number. In addition, the continuum wave function becomes more compact with increasing energy, that is, its nodes move in towards the nucleus. In the complicated overlap of positive and negative amplitudes which go into the dipole matrix element, it may be that at some energy the positive and negative components just cancel giving a zero in the dipole matrix element [11]. This is the reason behind the appearance of a Cooper minimum in photoionization cross section curves. It was found from the earlier study [11] that these Cooper minima are extensively pervasive and at least one sub-shell is found to have one minimum for all $Z>0$, and some high $Z$ elements may have many $[12,13]$. The data for all the alkali metal vapors, with the exception of lithium, show such minima in the cross-section curves close to ionization threshold. For lithium, photoionization cross section for $n s$ states has a maximum near threshold and then drop off monotonically [27]. To this end plasma screening effect has been studied. In particular, we chose the 2 seconds state of lithium to illustrate the result that a Cooper minimum can occur in the photoionization of the ground state of $\mathrm{Li}$ in a plasma environments, as well as to point out some implications of the plasma screening on this minimum.

Calculations have been performed by complex coordinate rotation method which employs square-integrable basis functions for computation of photoionization cross sections. Details regarding the basic theory and computational procedure have been discussed elsewhere [24-26, 28]. However, the theoretical studies of photoionization of multi-electron systems face some major difficulties because the photoionization calculations require estimates of the wave functions of the atomic system which are accurate in the regions of electron configuration space relevant to the calculations. In practice, assumptions have to make in order to obtain the necessary wave function. In the present calculation we employ a simple model potential of the form

$$
V_{m}(r)=-\frac{1}{r}\left[\left(Z-N_{c}\right)+N_{c}\left(e^{-2 \alpha r}+\beta r e^{-\gamma r}\right)\right] \text {, }
$$

to represent the interaction between the valence electron and the ion core. Where $\alpha, \beta$, and $\gamma$ are the model parameters, $Z$ is the nuclear charge and $N_{c}$ is the number of electrons in the core shell. In this approximation, which has been shown to give quite reasonable results for ground state atoms, the photoionization cross section is given by

$$
\sigma(\omega)=4 \pi \omega c^{-1} \theta^{3 N} \operatorname{Im} \sum_{i} \frac{\left[\bar{\psi}_{i}(r)|\mu(r \theta)| \psi_{0}(r \theta)\right]^{2}}{E_{i}-E_{0}-\omega}
$$

where $N$ is the number of electrons in the system, $\omega$ is the photon frequency, and $\mu$ is the dipole operator in the direction of the electric field. The basis function referring to $\psi_{0}$ contains complex coordinates while $\psi_{i}$ are linear combination of the un-rotated basis. The numerical accuracy of our work can be gauged by a comparison of cross sections computed for hydrogen, helium, lithium and sodium. The

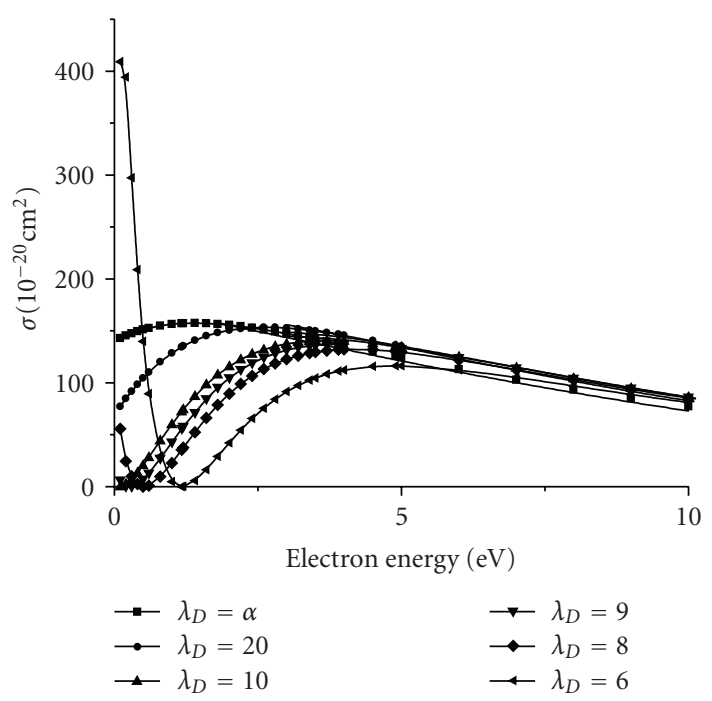

FIgure 1: Photoionization cross section $(\sigma)$ curve for Li ground statefor different Debye screening $\left(\lambda_{D}\right.$ in a.u.) versus ejected photoelectronenergy.

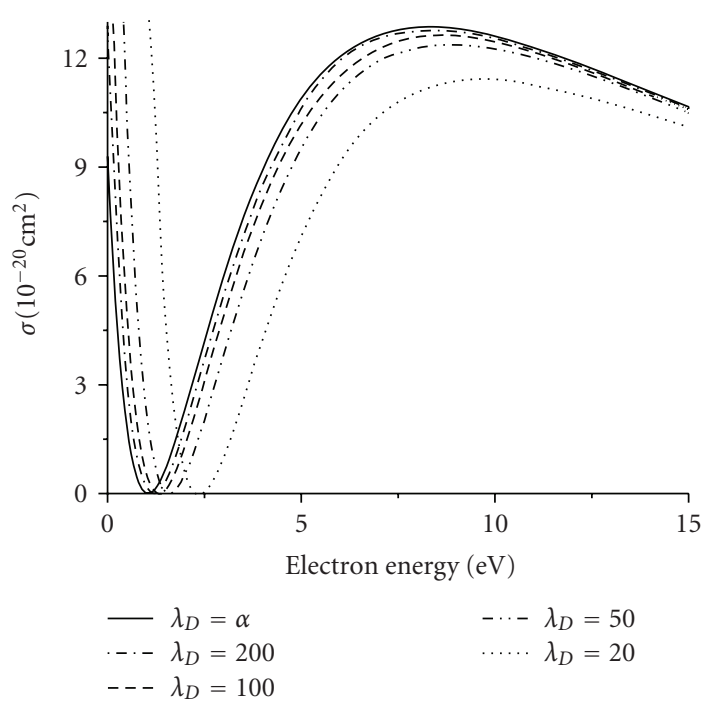

FIGURE 2: Photoionization cross section of the sodium ground state for different Debye screening as a function of the ejected photoelectron energy. $\lambda_{D}$ is the Debye screening length in a.u.).

results of our calculation [24-26] agree reasonably well with other theoretical and experimental results. The plasma screening effect has been investigated in Debye-Hückel model. In our previous work [26], we have examined the effects of plasma screening on photoionization of $\mathrm{Li}$ and $\mathrm{Na}$ from the ground state and have shown that photoionization is very sensitive to the plasma screening near the ionization threshold.

The results of the plasma screening effect on the photoionization cross-sections of $\mathrm{Li}$ and $\mathrm{Na}$ ground state are depicted in Figures 1 and 2, respectively. The strong screening remarkably changes the photoionization cross sections near the threshold. It is observed that the threshold cross section 
TABLE 1: Ground state energy ( $E_{g}$ in a.u.) and photoelectron energy ( $\varepsilon$ in a.u.) at Cooper minimum of Li and Na for different Debye screening length $\lambda_{D}$ (a.u.).

\begin{tabular}{|c|c|c|c|c|c|}
\hline \multicolumn{3}{|c|}{$\mathrm{Li}$} & \multicolumn{3}{|c|}{$\mathrm{Na}$} \\
\hline$\lambda_{D}$ & $\varepsilon$ & $E_{g}$ & $\lambda_{D}$ & $\varepsilon$ & $E_{g}$ \\
\hline 2.0 & - & No bound state & 2.0 & - & No bound state \\
\hline 3.0 & 0.101097 & -0.013282 & 3.0 & 0.295830 & -0.006226 \\
\hline 4.0 & 0.079121 & -0.036019 & 4.0 & 0.235561 & -0.025245 \\
\hline 5.0 & 0.059276 & -0.056043 & 5.0 & 0.204692 & -0.043900 \\
\hline 6.0 & 0.042996 & -0.072380 & 6.0 & 0.183378 & -0.059747 \\
\hline 7.0 & 0.029767 & -0.085633 & 7.0 & 0.167208 & -0.072873 \\
\hline 8.0 & 0.018962 & -0.096491 & 8.0 & 0.154713 & -0.083763 \\
\hline 9.0 & 0.009959 & -0.105505 & 9.0 & 0.144056 & -0.092881 \\
\hline 10.0 & 0.002425 & -0.113089 & 10.0 & 0.135604 & -0.100596 \\
\hline 11.0 & No Min. & -0.119547 & 20.0 & 0.093342 & -0.140253 \\
\hline 20.0 & No Min. & -0.178471 & 50.0 & 0.064678 & -0.168256 \\
\hline 100.0 & No Min. & -0.188122 & 100.0 & 0.054388 & -0.178355 \\
\hline 200.0 & No Min. & -0.193084 & 200.0 & 0.049244 & -0.183555 \\
\hline$\alpha$ & No Min. & -0.198141 & $\alpha$ & 0.044099 & -0.188857 \\
\hline
\end{tabular}

for Li is gradually reduced due to the plasma screening. The outstanding feature of this curve is the rich structure in evidence as a function of Debye screening length $\left(\lambda_{D}\right)$. Interestingly, a Cooper minimum starts to appear in the photoionization cross-section curve of Li when $\lambda_{D}<\lambda_{D}^{0}$, $\lambda_{D}^{0}$ is the Debye screening length at which threshold crosssection becomes zero. This is a completely new phenomenon, which is not observed so far. Over the past few decades our understanding of the atomic photoionization process has increased very much. It is known for some time that these minima can occur for all outer and near-outer subshell whose wave functions have nodes, except for 2 seconds [11]. The existence of Cooper minimum in photoionization cross section of the ground state of atom $(\mathrm{Na})$ has been tested experimentally [29]. Furthermore, we observe that the location of Cooper minimum in the photoionization cross section curve shifts toward the higher photon energy as the plasma screening increases (see Figures 1 and 2). In Table 1, we list the approximate positions of the Cooper minimum at different Debye screening length. The spectral shape of the cross sections away from threshold can be understood by considering the energy dependence of the dipole matrix element. To account for the appearance of Cooper minimum, as discussed in previous studies, the deviations of the wave functions from hydrogenic are required to produce zeros (since hydrogen never shows zero in any case of photoionization) and the relative phase difference characterized by the continuum wave-functions and the discrete states becomes deterministic. Qualitatively, the effect can be described this way. An ion exerts electric field on any atom or ion it passes. This electric field polarizes the latter, and spoils its spherical symmetric free space environment. Thus plasma environment effectively introduces a phase shift, which characterizes and provides a very sensitive measure of the non-Coulombic part of the eigenenergy, now becomes important to account for the zeros in photoionization cross section curve. This point can be understood in simple terms that the continuum phase shift is a function of electron energy, depending on its $l$-value. Accordingly, $p$-wave phase shifts decrease monotonically from threshold for all $\varepsilon$ [30]. Furthermore, the plasma environment introduces instability to the bound states, as it is evident from the fact that the energy of the ground state increases as Debye screening increases. The increased energy results in the electron being on the average pulled further away from the attractive force of the nucleus. As a result, nodes appear in the probability distribution of the electron. The number of nodes increases with increasing the plasma screening.

For isolated $\mathrm{Na}$, the relative phase difference between the bound 3 seconds state and the threshold function is just about $\pi / 2$. Thus a zero is expected in the 3 seconds $\rightarrow \varepsilon p$ matrix element near threshold. But for Li (2 seconds), which has no Cooper minimum for the free atom (no plasmas effect), the relative phase difference is only about $0.3 \pi$, which is too small to cause a zero [30]. This effect, however, comes as a surprise in plasma environment. It appears that the contribution of the inner core is probably quite significant in this case. The presence of plasma around the atom introduces the outer screening effect on the electron. The outer screening effect represented by the outer screening potential depends on the number and radial distribution of the outer electrons (ions) around the atom and this presence makes itself felt when plasma screening is sufficiently large. As expected this modification results in lowering of the binding energy, corresponding to increase the instability of the atom which is embedded in Debye plasma. The introduction of the outer screening potential induced by the plasma environment implies that the threshold for photoionization of electrons of a given shell is lowered by the corresponding value of the screening potential. The plasma screening effect is substantially decreased with an increase of Debye screening length. When the Debye screening length is sufficiently large, the outer screening has no appreciable effect on the photoionization spectrum. Thereby the outer 
screening effect influences the spectrum of photoionization cross sections only to the extent that it allows contributions to occur in spectral ranges. As a first step in understanding the reason for the appearance of Cooper minimum in photoionization of Li ground state in plasma environment note that, the dipole matrix elements can be divided into two parts-the first is the contribution of the outer region where the potential has gone asymptotic and is purely Coulomb and the second is the contribution of the inner region where the potential is completely non-Coulombic. Although the contribution from both of this region to the matrix element is important for the appearance for the Cooper minimum, we consider only the effect of the outer region as a useful zeroth approximation. For a large Debye screening length at least, the contributions to the dipole matrix elements come almost entirely from the large- $r$ region where the potential is Coulombic. Thus the wave functions, both discrete and continuum are hydrogenic in this region, except for a phase shift generated by the small non-Coulomb region near the nucleus. It is evident from the Figures 3(a) and 4(a) that the ionization threshold decreases monotonically when the Debye screening $\left(\lambda_{D}^{-1}\right)$ increases. The representation of the effect of the outer screening by the parameter $\lambda_{D}^{-1}$ influences the energy eigenvalue and the wavelength of the discrete or continuum wave-functions in the range of $r$ that contributes primarily to the dipole matrix element. More specifically, it affords control over the position of the nodes and the maxima of discrete and continuum wave-functions in this range, a factor that has great influence on the appearance of the Cooper minimum in the spectrum of photoionization cross sections. Mechanistically, one may think of resolving the process of photoionization into two stages, namely the photoionization which takes place in the region of space occupied by the initial orbital and the subsequent escape of the excited electron from this region. The process of escape through the outer plasma environment can modify the probability of photoionization. As the knowledge gathered from the quantum defect theory, for the electrons in the valence shell and in excited states in the presence of outer electrons, the inner screening of the nucleus adjusts itself to yield realistic energy eigen-values and thus make a wave function more realistic by adjusting its phase of oscillation. So, the phase shift [31] of the bound state introduced by the plasma decreases with the Debye screening length and thereby, the relative phase difference between zero energy continuum phase shift and the phase shift of the ground state induced by the non Coulombic inner core is gradually increased by plasma environment. This will cause a decrease in threshold cross sections as the positive and negative amplitudes of the bound and continuum states in dipole matrix elements overlap according to their phase difference, and they almost completely cancel each other when a relative phase shift of about $\pi / 2$ is introduced by sufficient plasma screening. As a result, a zero occurs near the threshold when $\lambda_{D}$ is reduced to $\lambda_{D}^{0}$. It must be emphasized, further that as plasma screening increases, the interaction of the inner core gradually becomes important. As a result, discrete wave function becomes quite diffuse and its nodes move toward the nucleus. Thus the situation arises that exact nodal

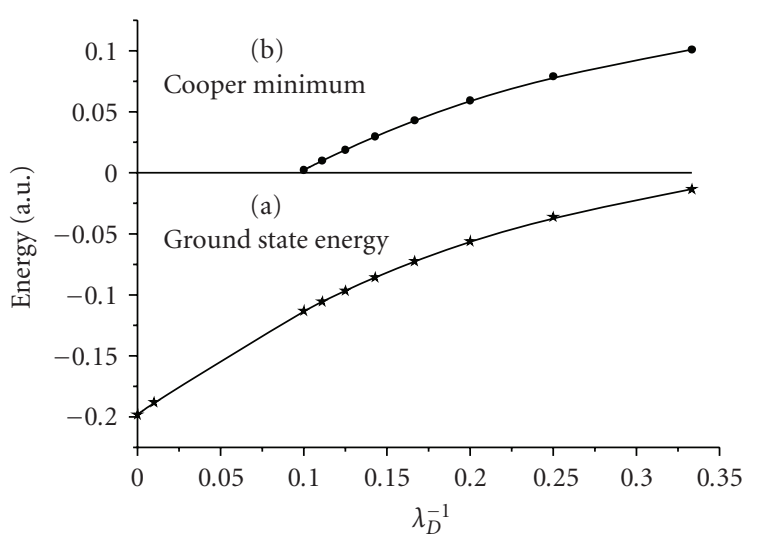

Figure 3: Location of Cooper minimum in photoionization cross section curve for Li ground state (in photoelectron energy in a.u.) and ground state energy (a.u.) versus Debye screening parameter $\left(\lambda_{D}^{-1}\right.$ in a.u. $\left.{ }^{-1}\right)$.

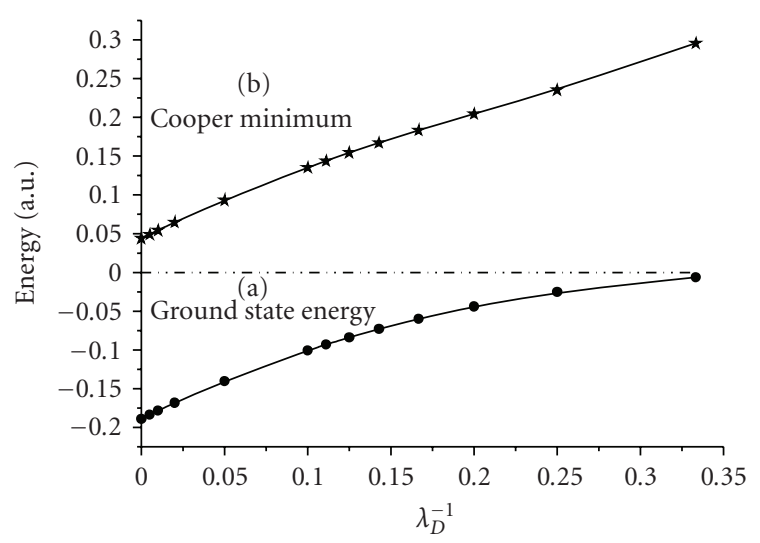

FIGURE 4: Location of Cooper minimum in the photoionization cross section curve for $\mathrm{Na}$ ground state in photoelectron energy in a.u. and ground state energy (a.u.) versus Debye screening parameter $\left(\lambda_{D}^{-1}\right.$ in a.u. $\left.{ }^{-1}\right)$.

shift of 2 seconds at $\lambda_{D}^{0}$ is just above what is needed for complete cancellation of the dipole matrix element with $\varepsilon p$ at threshold. The same analysis applies to the shift of Cooper minimum toward the higher energy region (Figures 3(b) and $4(\mathrm{~b}))$ as the plasma screening increases. Decreasing $\lambda_{D}$ below $\lambda_{D}^{0}$, the quantum defect for the bound state decreases the phase shift more than $\varepsilon p$ threshold phase shift. Consequently the relative phase difference increases so that the zero occurs at higher energy where $\varepsilon \mathrm{p}$ has the right value for correct overlap.

Several important points have emerged from this study. The photoionization is very sensitive to the plasma screening near the ionization threshold. The location of Cooper minima in ground states of atom ( $\mathrm{Li}$ and $\mathrm{Na}$ ) in Debye plasma has been calculated over the entire range of $\lambda_{D}$ (Debye screening length), and their behavior as a function of $\lambda_{D}$, has been explained primarily in terms of the instability of the bound states and the phase shifts of the discrete and the continuum states of the atom in the plasma environments. 
It is seen that a zero appears in the photoionization cross sections of Li in plasma environments when sufficient plasma screening effect modifies the core interaction in such a way that dipole matrix elements interfere destructively. It is also seen that with increasing plasma screening, the Cooper minima move further from thresholds owing to the increasing diffuseness of the ground states of the atoms. Since the screening effect, in one form or the other, is a common phenomenon in weakly coupled plasmas, the present results based strictly on the validity of the Debye model, exhibit some general and qualitative features.

\section{Acknowledgment}

The financial support by the National Science Council of Taiwan is gratefully acknowledged.

\section{References}

[1] R. W. Ditchburn, J. Tunstead, and J. G. Yates, "The continuous absorption of light in potassium vapour," Proceedings of the Royal Society A, vol. 181, pp. 386-399, 1943.

[2] D. R. Bates, "The quantal calculation of the photo-ionization cross-section of atomic potassium," Proceedings of the Royal Society A, vol. 188, pp. 350-357, 1947.

[3] M. J. Seaton, "A comparison of theory and experiment for photo-ionization cross-section. II. Sodium and alkali metals," Proceedings of the Royal Society A, vol. 208, pp. 418-430, 1951.

[4] J. W. Cooper, "Photoionization from outer atomic subshells. A model study," Physical Review, vol. 128, no. 2, pp. 681-693, 1962.

[5] J. W. Cooper, "Interaction of maxima in the absorption of soft X rays," Physical Review Letters, vol. 13, no. 25, pp. 762-764, 1964.

[6] E. J. McGuire, "Atomic photo-ionization cross sections from a semiempirical central potential," Physical Review, vol. 161, no. 1, pp. 51-59, 1967.

[7] E. J. McGuire, "Photo-ionization cross sections of the elements helium to xenon," Physical Review, vol. 175, no. 1, pp. 20-30, 1968.

[8] R. L. Smith and R. W. Labahn, "Photoionization of sodium, lithium, and potassium by a pseudopotential method," Physical Review A, vol. 2, no. 6, pp. 2317-2324, 1970.

[9] J. C. Weishiet, "Photoabsorption by ground-state alkali-metal atoms," Physical Review A, vol. 5, pp. 1621-1630, 1972.

[10] J.-J. Chang and H. P. Kelly, "Photoabsorption of the neutral sodium atom: a many-body calculation," Physical Review A, vol. 12, no. 1, pp. 92-98, 1975.

[11] S. T. Manson, "Systematics of zeros in dipole matrix elements for photoionizing transitions: nonrelativistic calculations," Physical Review A, vol. 31, no. 6, pp. 3698-3703, 1985.

[12] A. Z. Msezane and S. T. Manson, "Generality and systematics of multiple minima in photoionization cross sections of excited atoms," Physical Review Letters, vol. 48, no. 7, pp. 473$475,1982$.

[13] J. Lahiri and S. T. Manson, "Multiple minima in photoionization cross sections of excited atoms," Physical Review Letters, vol. 48, no. 9, pp. 614-616, 1982.

[14] J. H. Hoogenraad and L. D. Noordam, "Rydberg atoms in farinfrared radiation fields. I. Dipole matrix elements of $\mathrm{H}, \mathrm{Li}$, and Rb," Physical Review A, vol. 57, no. 6, pp. 4533-4545, 1998.
[15] Y.-D. Jung, "Plasma-screening effects on the electron-impact excitation of hydrogenic ions in dense plasmas," Physics of Fluids B, vol. 5, no. 9, pp. 3432-3440, 1993.

[16] Y.-D. Jung, "Semiclassical transition probabilities for the electron-impact excitation of hydrogenic ions in dense plasmas," Physics of Plasmas, vol. 2, no. 1, pp. 332-336, 1995.

[17] Y.-D. Jung, "Oriented s-p excitation in electron-ion collisions in dense plasmas," Physics of Plasmas, vol. 2, no. 3, pp. 987990, 1995.

[18] Y.-D. Jung, "Plasma screening effects on photoionizations in weakly coupled plasmas," Physics of Plasmas, vol. 5, no. 10, pp. 3781-3785, 1998.

[19] Y.-D. Jung, "K-shell photoionizations in strongly coupled plasmas," Physics of Plasmas, vol. 5, no. 12, pp. 4456-4460, 1998.

[20] W. Hong and Y.-D. Jung, "Plasma-screening effects on direct $1 s \rightarrow 2 p_{ \pm}$electron-ion collisional excitation rates in dense plasmas," Physics of Plasmas, vol. 3, no. 6, pp. 2457-2460, 1996.

[21] J.-S. Yoon and Y.-D. Jung, "Spherical versus nonspherical plasma-screening effects on semiclassical electron-ion collisional excitations in weakly coupled plasmas," Physics of Plasmas, vol. 3, no. 9, pp. 3291-3296, 1996.

[22] C.-G. Kim and Y.-D. Jung, "Semiclassical electron capture probabilities for proton-hydrogenic ion collisions in dense plasmas," Physics of Plasmas, vol. 5, no. 7, pp. 2806-2809, 1998.

[23] C.-G. Kim and Y.-D. Jung, "Dynamic plasma screening effects on semiclassical electron captures from hydrogenic ions by protons in weakly coupled plasmas," Physics of Plasmas, vol. 5, no. 10, pp. 3493-3496, 1998.

[24] L. B. Zhao and Y. K. Ho, "Influence of plasma environments on photoionization of atoms," Physics of Plasmas, vol. 11, no. 4, pp. 1695-1700, 2004.

[25] A. C. H. Yu and Y. K. Ho, "Stark shifts and widths of a hydrogen atom in Debye plasma," Physics of Plasmas, vol. 12, no. 4, Article ID 043302, 2005.

[26] S. Sahoo and Y. K. Ho, "Photoionization of Li and $\mathrm{Na}$ in plasma environments," Physics of Plasmas, vol. 13, no. 6, Article ID 063301, 2006.

[27] G. Peach, H. E. Saraph, and M. J. Seaton, "Atomic data for opacity calculations. IX. The lithium isoelectronic sequence," Journal of Physics B, vol. 21, no. 22, pp. 3669-3683, 1988.

[28] T. N. Rescigno and V. McKoy, "Rigorous method for computing photoabsorption cross sections from a basis-set expansion," Physical Review A, vol. 12, no. 2, pp. 522-525, 1975.

[29] R. D. Hudson and V. L. Carter, Journal of the Optical Society of America, vol. 57, p. 651, 1967.

[30] S. T. Manson, "Dependence of the phase shift on energy and atomic number for electron scattering by atomic fields," Physical Review, vol. 182, no. 1, pp. 97-103, 1969.

[31] L. Rosenberg, "Levinson-Seaton theorem for potentials with an attractive Coulomb tail," Physical Review A, vol. 52, no. 5, pp. 3824-3826, 1995. 

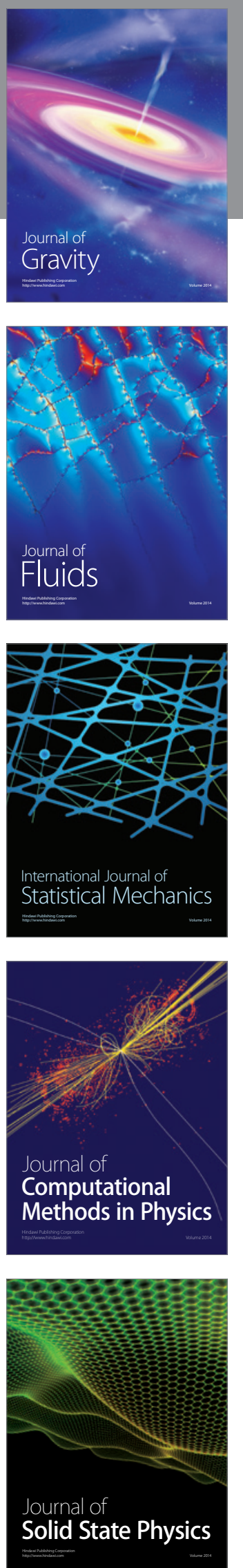

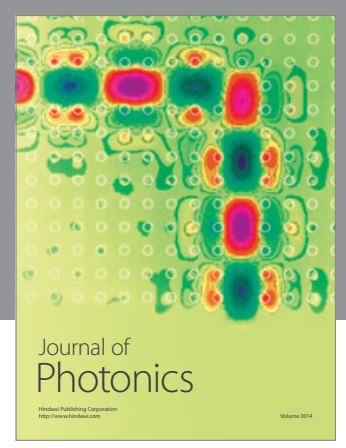

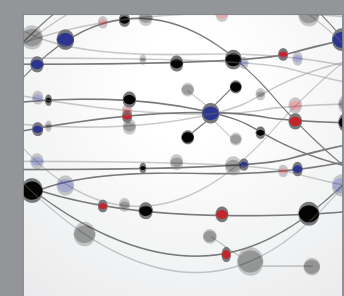

The Scientific World Journal
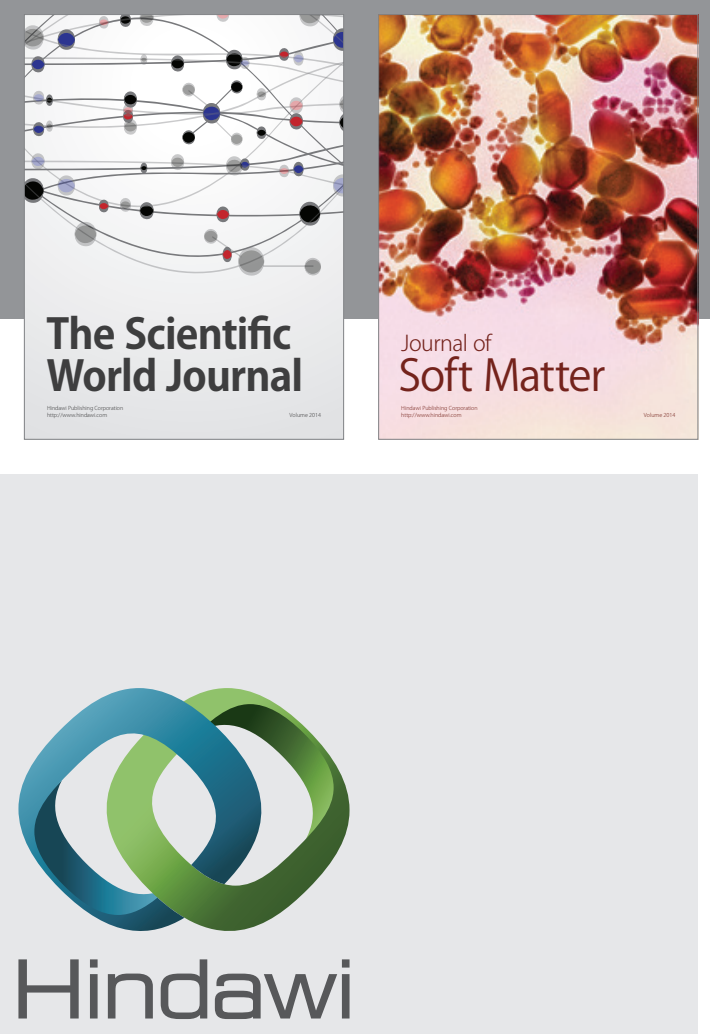

Submit your manuscripts at

http://www.hindawi.com
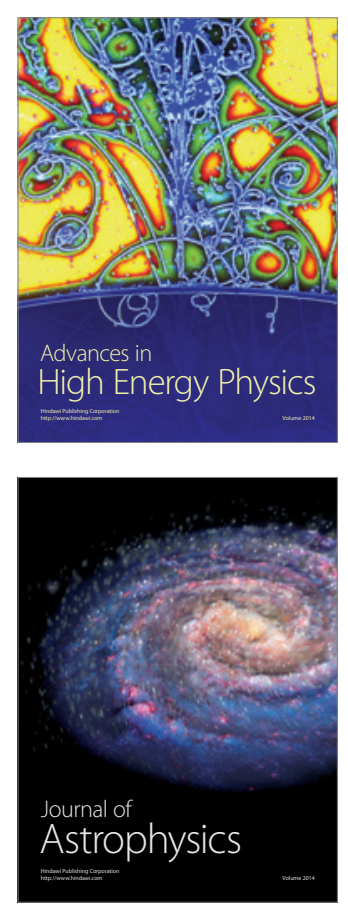
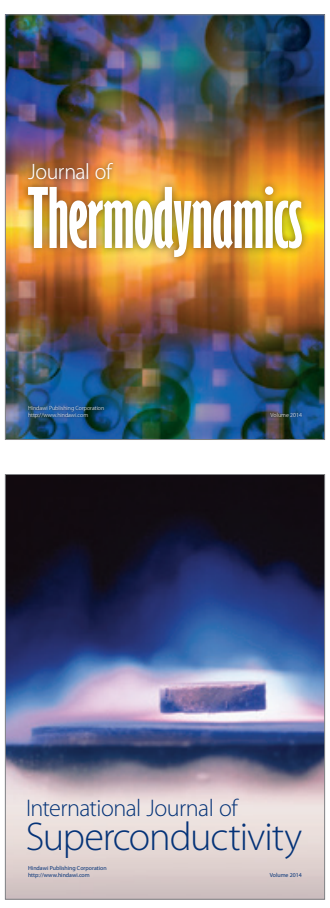
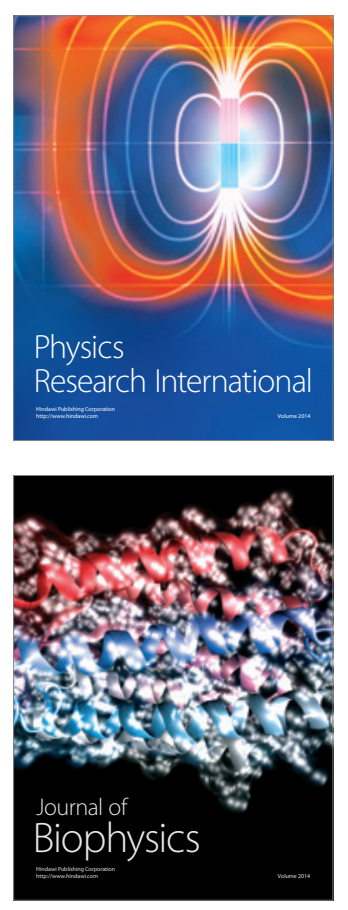
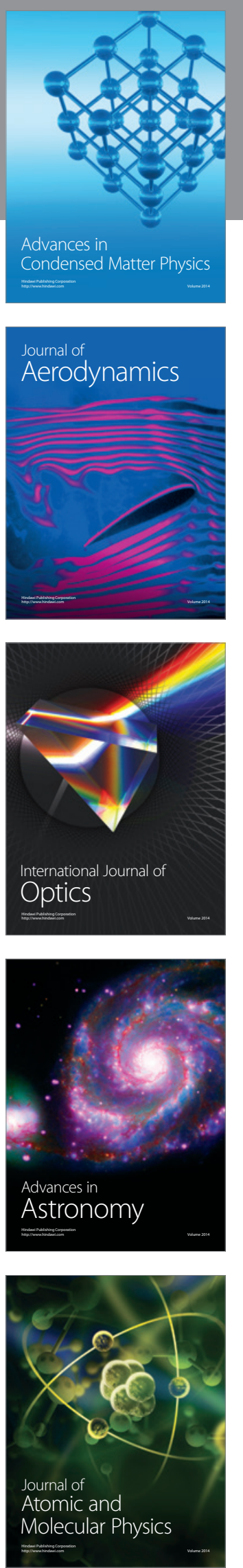